\title{
Bayesian Learning of the Correlation Structure of High-Dimensional Random Objects.
}

\author{
Dr. Dalia Chakrabarty \\ Loughborough University, UK \\ D.Chakrabarty@lboro.ac.uk
}

\begin{abstract}
One of the fundamental challenges in contemporary Statistics is the learning of the correlation structure of a high-dimensional random variable, the different elements of which are differently correlated, and realisations of which are discontinuously distributed. Parametric modelling of such correlation is then successful only when hyper-parameters of the invoked parametric correlation kernels, are modelled to adapt to the discontinuity in the data. I will show that this requirement leads to a 2-layered learning strategy described by the compounding of a tensor-variate stochastic process with multiple scalar-variate stochastic processes. I will also talk about a new way of modelling the absolute correlation between a pair of multivariate data sets, where this correlation is informed on, by a new metric that computes the distance between the posteriors of the graphical models learnt given the data pair, where such graph learning is extended to learn large networks. The discussed methods will be empirically illustrated on real data.
\end{abstract}

